\title{
Fertility Outcome of Surgical Recanalization of Vas Deferens in Individuals with Non Scalpel Vasectomy (NSV)
}

\author{
HR ROY $^{\mathrm{a}}$, N ISLAM ${ }^{\mathrm{b}}$, SMS ISLAM ${ }^{\mathrm{c}}$
}

\begin{abstract}
:
Background: Non Scalpel Vasectomy (NSV) is being widely used by Family Planning Sector of Bangladesh for male sterilization. But when young persons with NSV needs reversal of the procedure (recanalization), they become frustrated hugely affecting the family harmony.

Aims and Objectives: To see the efficacy of recanalization of Vas Deferens of Non Scalpel Vasectomy (NSV) Individuals. To establish confidence on national NSV program by assuring the scope of future reversal if needed.

Materials and methods: During the period of January 2017 to November 2019, we have analyzed the outcome of recanalization surgery of all cases of NSV individual which were referred to our urology team at Rangpur of Bangladesh. We excised the involved segment of Vas Deferens and stented it internally by No-1 prolene (Ethicon). Then end-to-end anastomoses were done by $7 / 0$ vicryl (Ethicon). We collected articles from Hinari, Pubmed and Banglajol with extensive
\end{abstract}

Introduction:

Vasectomy for male sterilization for family planning is an established permanent method. No Scalpel Vasectomy (NSV) is being widely practiced by Family Planning sector of Bangladesh. Each Family Planning Centre of Bangladesh Govt. at Upazila level are doing lots of NSV as a national program. Father of two or more children with eligible couple are being selected randomly for NSV.

But some new problems are being fetched with this national NSV program. Two factors are responsible: 1) Death of child of eligible NSV client, 2) Divorce or death

1. Dr. Hriday Ranjan Roy, Associate Professor of Surgery, Rangpur Medical College, Rangpur, Bangladesh

2. Dr. Nabiul Islam, Medical Officer, MCH-FP, Rangpur, Bangladesh

3. SM Saidul Islam, Deputy Director, Family Planning, Rangpur, Bangladesh

Address of Correspondence: Dr. Hriday Ranjan Roy, Associate Professor, Department of Surgery, Rangpur Medical College, Rangpur, Bangladesh. Mobile: 01712841244, Email: hriday.roy@gmail.com

Received: 19 January, 2020

Accepted: 24 March, 2021 searches. We have compared our findings to the previously published articles.

Result- We found $91.9 \%$ success rate in this study with positive semen production. Pregnancies were achieved in wives of $72.9 \%$ of recanalized individuals. The most common causes of unsuccessful recanalizations are more than 7 years of NSV, knot in close proximity to epididymis, two or more knots and cord like Vas Deferens. One patient had significant hematoma leading to a failed outcome, otherwise there was no mentionable complication in this series.

Conclusion-Recanalization of Vas Deferens of NSV individuals can be done with high success rate and other centers of Bangladesh may take steps for further evaluation.

Key Words: Non Scalpel Vasectomy (NSV), Vas Deferens, Recanalization, Male Fertility, Male Sterilization.

(J Bangladesh Coll Phys Surg 2021; 39: 167-170)

DOI: https://doi.org/10.3329/jbcps.v39i3.54159

of wife with new marriage. In both the conditions, the young persons with NSV become frustrated and hugely affect the family harmony. Therefore, many vasectomized clients are eagerly searching for reversal procedures. But no national center or way are available. Moreover, many individuals inquire about the possibility of future recanalization and restoration of fertility. The traditional reply to this question has been that it is difficult to "reverse" NSV and that the success rate of reversal procedure is so small than the operation (NSV), for all practical purposes, should be considered permanent and irreversible ${ }^{1}$.

The psychological anxiety of the vasectomized person is enormous and failure to reproduce in situations of need creates a social distance for the Family Planning personnel which may lead to limit the acceptability of NSV. Moreover, spreading social anxiety influences the family planning program negatively. The traditional views have been persisted in spite of the many reports of highly successful vas recanalizations during last few years ${ }^{1}$. Early studies of surgical recanalizations reported success rate in about $35 \%$ to $45 \%$ of cases ${ }^{2-4}$. More recently, it has been shown that it is possible to attain recanalization in as many as $70 \%$ to $80 \%$ of cases ${ }^{5-7}$. 
In Bangladesh, an article was published in daily newspaper (The Daily Nayadigonta, 21 Oct. 2017) where success rate were shown to be $75 \%$ and $45 \%$ respectively. But these findings (study) were not conducted by the author himself. Extensive search revealed no article published from Bangladesh in any recognized medical journal.

\section{Materials and methods:}

During the period of January 2017 to November 2019, 45 individuals with previous NSV of were included into this study. Candidates for surgical recanalization were selected in collaboration with Family Planning department of Rangpur Division. Operations were done in Rangpur Medical College Hospital, Life Line Hospital and New Rangpur Clinic of Rangpur Bangladesh. All epidemiological and operative procedures, complication and outcome related data were saved in a registers. Preoperative and post-operative semen analysis, follow up for having pregnancies of their wives were also recorded. Any disease of testis or scrotum (hydrocele, pyocele, testicular atrophy, flabby on palpation etc) were excluded from the study. Appearance of sperms in semen of previously azoospermic patients were considered as success. All records were saved properly. Informed consents were taken from all individuals regarding operative procedure and outcome.

\section{Operative Technique:}

All operations were done under spinal anesthesia. After excluding any other comorbidity all individuals were evaluated for operative fitness. Physical examination of scrotum was done routinely to evaluate the testes especially to locate the knot of NSV. The knot should be at least one inch above the upper pole of testis. If any suspicion arise regarding condition of testis, an ultrasonogram was done.

After exploration of the vas, affected segment was excised as less as possible. Close proximity of double knot could be excised in Toto. In 2 cases, we found double knots more than one inch apart. In those cases, we excised knots separately keeping normal segment in situ to expose lumen of vas. In 3 cases, we found a gap between two ends of vas having a small pocket full of semen like materials. In these cases, we found presence of abnormal reports of semen analysis preoperatively. Isolation of vas and trimming of both ends were done to expose lumen. Then we passed a No. 1 prolene proximally up to prostate or as far as possible, through intervening normal segment (in 3 cases) and distally up to upper pole of testis. Distally, we give a tiny incision to the vas to take out distal end of prolene. This acts as a stent, ensures patency \& continuity of lumen and guide anastomosis by $7 / 0$ vicryl. After completing anastomoses, the lower end of prolene was taken outside through a tiny hole of scrotal skin. Then it was fixed to the scrotal skin and wound was closed. After completing of opposite side, both ends were tied together and fixed. Otherwise, it may be lost and enter into vas which we found in one case. A light coconut bandage was applied. Stitches and prolene were removed after 7 days of operation. A repeat semen analysis was done after one month with advice of abstinence for 7 days after operation.

\section{Results:}

During the study period total 45 individuals who had NSV in the past reported to us for recanalization. Eight patients were excluded from the study because of presence of different exclusion criteria. Finally 37 persons were included in this study. After the surgical procedure, out of these 37, 34 were found sperm positive which accounts $91.9 \%$ of success rate. Among 34 cases, 27 individuals $(72.9 \%)$ later informed us about conception of their wives. Among three unsuccessful cases, one had atrophied testes, one had severe hematoma formation postoperatively and one had the NSV knot very close to the testis $(2 \mathrm{~cm})$.

Recanalization were done within three years in 10 persons, five years in 19 and seven years in seven individuals respectively. In one individual, who had NSV eight years back, the recanalization was unsuccessful.

In one individual, we found the distal NSV knot very close to the testis $(2 \mathrm{~cm})$ involving convoluted epididymis which was also unsuccessful. In 2 cases we anastomosed vas to epididymis and were sperm positive after operation.

In one case, we found multiple knots with cord like appearance of Vas and involve more than one inch of segment. This patient also had postoperative hematoma and was unsuccessful. But in another four cases, long segment involvements were found and operations were done satisfactorily with postoperative sperm positive. In one case, the two NSV knots were found more than one inch apart with intervening normal vas segment. 
We excised the knots separately keeping intervening Vas in situ, stented and two anastomoses were done on the same side. This case was also successful.

In two cases, we did not found any knot. Rather a gap was noted between the two ends. After exploration, we found a small pocket full of whitish material (semen). In these cases, we found oligospermia with abnormal sperm and pus cells on preoperative semen analysis. So this pocket could be considered as a stagnant station causing harm to sperm and development of pus cells. Anastomosis of these two cases by our method was successful and semen reports were satisfactory.

We noticed post-operative complications of hematoma in one case, stent (prolene) missing in one case and stitch infections were found in 5 cases respectively.

\section{Discussion:}

Many suitable vasectomy candidates raised concerns about their ability to produce children if existing children die or in case of a separation in the present marriage which will call for remarrying ${ }^{6}$. Moreover, accidental death of their wives is causes of remarrying. The general belief is that if the man decides to remarry in future after vasectomy, it may become difficult to have a child or children with the new wife ${ }^{6}$. The common belief is that it is a permanent method and also limits sexual ability. These beliefs seriously affect the national NSV program and limiting the success of the family planning project. So our study was intended to overcome this situation by successful recanalization of vas deferens and thus assure the candidate that successful reversal is possible with high success rate. And thus it will positively influence the national NSV program.

Vasectomy (NSV) should no longer be considered an irreversible procedure ${ }^{1}$. Pardanani et al ${ }^{1}$ published an article of 20 unselected recanalization of which result of 17 were given. Of 17 clients, $13(76.5 \%)$ had successful recanalizations and 5 (29.4\%) wives became pregnant. In this study, a medical grade silastic tube was used to stent the Vas Deferens externally for the procedure and 17 cases were divided into two groups. In group 1(satisfactory), out of 13 patients $12(92.3 \%)$ showed successful in which 4 of their wives were pregnant. In group 2 (unsatisfactory), four were included of which one (25\%) was successful whose wife was pregnant. Thus overall success and pregnancy rate were $76.5 \%$ and $29.4 \%$ respectively. In our study, we did not make such groups and out of 37
NSV cases 34 (91.9\%) were successful and 27 (72.9\%) of their wives became pregnant. Of 34 successful cases 7 $(18.9 \%)$ of their wives did not become pregnant though sperm counts were satisfactory. The causes of failure were not clearly identified. But age ( $>50$ years) of individuals, anti-sperm antibody, female partner problems might have possibilities. So, there is clear better result found in our study. This better result indicates clear superiority of our technique over silastic tubing. Another cause of better result in our study may be that all of our cases were NSV clients rather than conventional open surgical vasectomy.

Pardanani et al ${ }^{1}$ declared unsatisfactory candidates in which very low and long segment $(>1.5 \mathrm{~cm})$ vasectomy. In our study, we also found too much difficulty in these cases but not absolutely impossible. We performed recanalizations satisfactorily even in $2.5 \mathrm{~cm}$ (1 inch) segment involvement. Even where there were double knots, we kept the intervening normal segment in situ with excision of fibrous nodular knots with double anastomosis at same side. Very low vasectomy is really difficult for recanalization. It exposes convoluted tubule of epididymis and makes anastomosis tough which we experienced in our study. So our recommendations are: to do NSV at least one inch above the upper pole of testis and to avoid double knots. Accidental spontaneous recanalization is a rare possibility; rather scope of future reversal surgery is more important.

Most of the studies 1-6 showed recanalization unsuccessful in cases of more than 7 years interval from vasectomy. We also found it unsuccessful in clients of recanalization who had NSV more than 7 years back. So it is a critical decision and matter of much concern and counselling in these cases and should be explained clearly to each individual. Though, counselling of no warranty should be told to every candidate of recanalization.

\section{Conflicts of Interest:}

Nothing to declare.

\section{Conclusion:}

Recanalization of Vas Deferens by this procedure found to be acceptable with higher success rate and other centers of Bangladesh may take steps for further study and evaluation. NSV candidates can be assured of future reversal if needed, this information will surely provide a positive impact on family planning (NSV) Campaign of Bangladesh. 


\section{References:}

1. Pardanani DS, Kothari ML, Pradhan SA, Mahendrakar MN: Surgical restoration of vas continuity after vasectomy: Further clinical evaluation of a new operation technique. Fertility and Sterility 1974; 25(4): 319-324.https://doi.org/ 10.1016/S0015-0282(16)40331-6

2. Cameron GS: Anastomosis of the vas deferens. JAMA 1945: 127(119). https://doi.org/10.1001/jama.1945.928601 70001007,PMid: 12275695

3. Dorsey JW: Anastomosis of vas deferens to correct postvasectomy sterility. J Urol 1953; 70(515).https://doi.org/ 10.1016/S0022-5347(17)67941-2

4. O'Connor VJ: Anastomosis of the vas deferens after purposeful division for sterility. JAMA 1948; 136(162). https://doi.org/10.1001/jama.1948. 02890200016004. PMid:18920081

5. Phadke GM, Phadke AG: Experiences in the re-anastomosis of the vas deferens.

6. Kar JK: Surgical correction of post-vasectomy sterility. J Family Welfare 1969; 15(50).

7. Jhaver PS: Conception and contraception. J Family Welfare 1962; 9(47).

8. Andogo PB, Tapsob P, Phillip JF et al: "If you do vasectomy and come back here weak, I will divorce you": a qualitative study of community perceptions about vasectomy in Southern Ghana. International Health and Human Rights 2014; 14(16): 1-8. ,https://doi.org/10.1186/1472-698X14-16 PMid:24885663 PMCid:PMC4019590 